\title{
Simulation of energetic particle interactions with a shock wave in a focused transport model
}

\author{
Yulia Kartavykh* \\ Institut für Theoretische Physik und Astrophysik, Universität Würzburg, 97074 Würzburg, \\ Germany; Ioffe Physical-Technical Institute, St. Petersburg 194021, Russia \\ E-mail: ykartavykheastro.uni-wuerzburg.de
}

\section{Wolfgang Dröge}

Institut für Theoretische Physik und Astrophysik, Universität Würzburg, 97074 Würzburg,

Germany

E-mail: droege@astro.uni-wuerzburg.de

\section{Michael Gedalin}

Department of Physics, Ben-Gurion Unversity of the Negev, Beer-Sheva, Israel

E-mail: gedalin@bgu.ac.il

\begin{abstract}
We use numerical solutions of the focused transport equation to study the evolution of the pitchangle dependent distribution function of protons in the vicinity of shock waves and compare the results with basic predictions of diffusive shock acceleration theory. We then consider the case that a seed population of protons is injected close to the Sun simultaneously with a traveling interplanetary shock for which we assume a simplified geometry. We investigate the effects of adiabatic focusing, pitch-angle dependent spatial diffusion, first-order Fermi acceleration at the shock and adiabatic energy losses in the expanding solar wind behind the shock. We analyze the resulting intensities, anisotropies, and energy spectra as a function of time and find that our simulations can reproduce the intensity-time profiles typically observed by the Helios, ACE and Wind spacecraft in interplanetary shock-associated particle events. The acceleration efficiency does not seem to be high enough to explain the energy spectra observed at the shock as being accelerated out of a thermal seed population during the time of $\sim 2$ days it takes the shock to reach the Earth, and an injection spectrum of particles pre-accelerated by solar flares or coronal shocks would be required.
\end{abstract}

The 34th International Cosmic Ray Conference,

30 July- 6 August, 2015

The Hague, The Netherlands

\footnotetext{
* Speaker.
} 


\section{Introduction}

Solar energetic particles events are typically divided into two classes - impulsive events with characteristic times for the observed fluxes of several hours, and gradual events, with fluxes lasting for several days [1]. Besides such characteristics, as enrichment by electrons, ${ }^{3} \mathrm{He}$, and charge states of heavy ions like iron, these classes are usually assessed to have different acceleration mechanisms. Particles in impulsive events are considered to be energized in solar flares by either stochastic acceleration and/or in a process involving magnetic reconnection. In gradual events particles are thought to be accelerated mainly at traveling interplanetary shock waves by the process of diffusive shock acceleration (DSA) [2] which requires a pitch angle distribution function of accelerated particles which is always close to isotropy. However, spacecraft observations in the past decades have shown that the fluxes in gradual SEP can be highly anisotropic, which makes the above consideration questionable. To address this problem a different approach was introduced $[3,4,5]$ by describing the transport of the particles by the focused transport equation, which is then solved with Monte-Carlo simulations of the corresponding stochastic differential equations (SDEs). This approach, which we will also employ in our work, does not require isotropic pitch angle distributions, allows relatively easily to incorporate the effect of shock acceleration without having to formulate matching conditions at the shock, and to investigate the effects of more complicated magnetic field structures.

\section{Pitch-angle dependent particle transport}

The evolution of the particle's distribution function $f(r, \mu, p, t)$, where $r$ is the location in the Heliosphere relative to the center of the Sun, $\mu=\cos \theta$ and $p$ are the particle's pitch angle cosine and momentum, respectively, and $t$ is the time, is determined by the following processes: adiabatic motion along the smooth diverging magnetic field, pitch angle scattering at magnetic irregularities, diffusion perpendicular to the magnetic field, and co-rotation. The pitch angle scattering gives rise to spatial diffusion along the field and tends to isotropize the distribution function. The latter effect also couples the particles to the expanding solar wind and leads to convection and energy losses due to adiabatic deceleration. Particle drifts perpendicular to the average field arise due to gradients and curvature in the field, and by the action of an induced electric field, $\boldsymbol{E}=\boldsymbol{V}_{s \boldsymbol{w}} \times \boldsymbol{B}$, where $\boldsymbol{V}_{s w}$ is the solar wind velocity and $\boldsymbol{B}$ denotes the magnetic field (co-rotation, [6]). A Fokker-Planck equation for $f(\boldsymbol{r}, \mu, p, t)$ which describes the above effects has been given by [7]. Note that $f(\boldsymbol{r}, p, \mu, t)$ is proportional to the measured flux or intensity of the particles, $I(\boldsymbol{r}, E, \mu, t)$, here formulated as function of the kinetic energy $E$, which is usually the observable. In this work we neglect co-rotation and the diffusion of the particles perpendicular to the magnetic field. We do consider the effect of first-order Fermi acceleration which the particles undergo if they encounter a shock wave and are being scattered forth and back through the velocity jump at the shock front. Here we consider only parallel shocks in which electric fields are absent.

Various techniques have been applied to numerically solve Fokker-Planck equations for the above effects. Monte-Carlo simulations of the corresponding Ito stochastic differential equations [8] were presented by [9] who used a time-backward stochastic process with an explicit scheme for the momentum transport, and by $[10,11,12]$ who used a time-forward stochastic process with 
an implicit scheme, which addresses energy-changing processes by performing proper Lorentz transformations between a stationary frame in which the Sun is at rest and frames which move with the momentary solar wind velocity at the position of the particle. Here we employ again the latter approach.

\section{Particle acceleration at a stationary parallel shock}

As a consistency check we compare results of our method with the predictions of DSA for a static shock front with different flow speeds on the each side of the shock front $\left(U_{1}\right.$ upstream and $U_{2}$ downstream in the shock's rest frame). In this part the remaining processes are advection along the magnetic field, scattering of particles at magnetic inhomogeneities, and convection with the flow. We assume a constant magnetic field, and therefore the particles are not subject to magnetic focusing. The remaining two stochastic differential equations to solve are then:

$$
d z(t)=\mu v d t \quad \text { and } \quad \mathrm{d} \mu(\mathrm{t})=\sqrt{2 \mathrm{D}_{\mu \mu}} \mathrm{dW}_{\mu}(\mathrm{t})+\frac{\partial \mathrm{D}_{\mu \mu}}{\partial \mu} \mathrm{dt}
$$

where $z$ is the coordinate along the magnetic field, $v$ the speed of th particles, $W_{\mu}(t)$ a onedimensional Wiener process, and $D_{\mu \mu}$ is the pitch angle diffusion coefficient. For comparison with diffusion-convection models for the isotropic part of the particle's distribution function we introduce a parallel mean free path

$$
\lambda_{\|}=\frac{3 v}{8} \int_{-1}^{+1} d \mu \frac{\left(1-\mu^{2}\right)^{2}}{D_{\mu \mu}(\mu)}
$$

which relates the pitch angle scattering rate to the spatial diffusion parallel to the ambient magnetic field. Figure 1 shows resulting energy spectra at different time intervals after the start of the proton injection for a shock with a compression ratio of $R=3$, for which DSA predicts a spectrum at the shock $\sim E^{-5 / 4}$ in the non-relativistic regime. It can be seen from the figure that our simulations asymptotically approach the above spectrum, on time scales which depend on the assumed values of the mean free path and the distances of the escape boundaries from the shock in the up- $\left(\lambda_{u}, z_{u}\right)$ and downstream $\left(\lambda_{d}, z_{d}\right)$ regions. In the left panel of the figure a size of the upstream region of 10 mean free paths was chosen, and a size of 70 mean free paths in the right panel. The downstream region had in both cases a length of 100 mean free paths. Not surprisingly, we find that increasing the size of the downstream region leads to an increase of the energy range where the modeled spectrum reproduces the prediction from diffusive shock acceleration, and that decreasing the values of $\lambda_{u}$ and $\lambda_{d}$ leads to a shorter time scale on which the resulting spectra approach the DSA predictions. We conclude that our simulations reproduce the basic features of DSA for a one-dimensional stationary shock correctly.

\section{Particle acceleration and transport at a traveling parallel interplanetary shock}

Now we consider transport and energy gain and loss processes of energetic protons in the vicinity of a shock wave propagating in the inner heliosphere. We assume that a seed population 

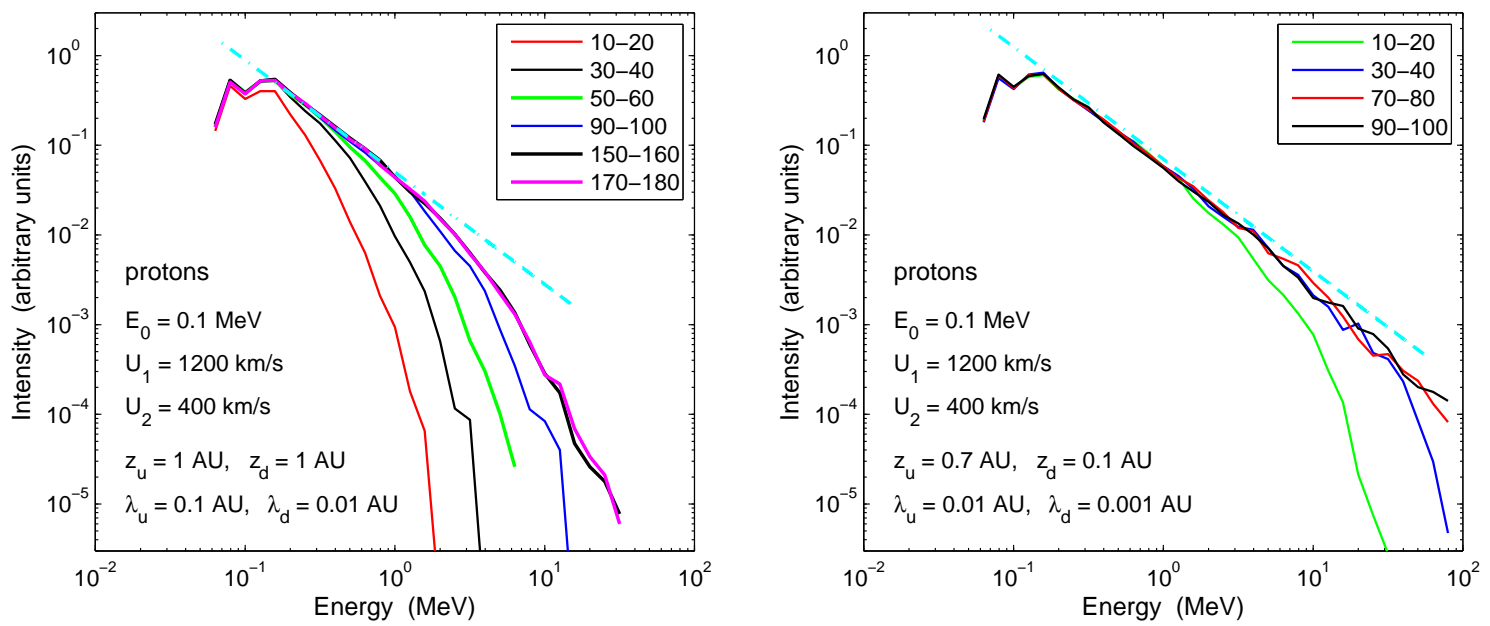

Figure 1: Energy spectra of particles at the shock with a compression ratio $R=3.0$ at different time intervals after the start of the injection (given on the legend in hours). Left panel: $\lambda_{u}=0.1 \mathrm{AU}, \lambda_{d}=0.01 \mathrm{AU}, z_{u}=1$ $\mathrm{AU}, z_{d}=1 \mathrm{AU}$. Right panel: $\lambda_{u}=0.01 \mathrm{AU}, \lambda_{d}=0.001 \mathrm{AU}, z_{u}=0.7 \mathrm{AU}, z_{d}=0.1 \mathrm{AU}$. Simulation results are given in the shock front system. The dash-dotted line indicates spectrum predicted by diffusive shock acceleration.

of particles is accelerated in a solar flare and is released into the interplanetary medium at a radial distance (with respect to the center of the Sun) $r_{0}$ simultaneously with a shock wave. For simplicity, we assume a radial interplanetary magnetic field (Fig. 2, upper left panel) and a spherically symmetric blast wave which propagates with a speed $V_{s h}$ into the undisturbed solar wind which is assumed to have a speed $V_{0}$. Under the above assumptions the shock is again strictly parallel. The solar wind speed relaxes behind the shock as a function of the radial distance $r$ linearly from a speed $V_{d}$ to $V_{0}$ at $r_{0}$ (shown schematically in Fig. 2, right panel). In the frame moving with the shock front the fluid velocity in the upstream region is then $U_{1}=V_{s h}-V_{0}$, and in the downstream region $U_{2}=V_{s h}-V_{d}$. The compression ratio at the shock is $R=U_{1} / U_{2}$. In this geometry the two stochastic differential equations adopt the form

$$
d r(t)=\mu v d t \quad \text { and } \quad \mathrm{d} \mu(\mathrm{t})=\sqrt{2 \mathrm{D}_{\mu \mu}} \mathrm{dW}_{\mu}(\mathrm{t})+\left[\frac{v}{2 \mathrm{~L}}\left(1-\mu^{2}\right)+\frac{\partial \mathrm{D}_{\mu \mu}}{\partial \mu}\right] \mathrm{dt}
$$

which now includes the effect of adiabatic focusing in a diverging magnetic field through the focusing length $L(r)=-B /(d B / d r)$. For the pitch angle diffusion coefficient $D_{\mu \mu}$ we adopt a product ansatz of the form

$$
D_{\mu \mu}(r, E, \mu)=\kappa_{0}(r, E) \cdot\left\{|\mu|^{q-1}+H\right\}\left(1-\mu^{2}\right)
$$

which partially resembles the result of standard quasi-linear theory [13] and additionally introduces a parameter $H$ which can phenomenologically describe an enhancement of scattering through $\mu=0$ by non-resonant and non-linear effects. The parameter $q$ denotes the spectral index of the magnetic fluctuations along the field, which is assumed to be a single power law in wave number. We adopt $H=0.5$ which is consistent with pitch angle distributions which are strongly forward peaked and proceed smoothly through $\mu=0$, as frequently observed for protons in the energy range considered here [14], and a value of $q=1.67$ for a Kolmogorov spectrum of the fluctuations. The solutions 

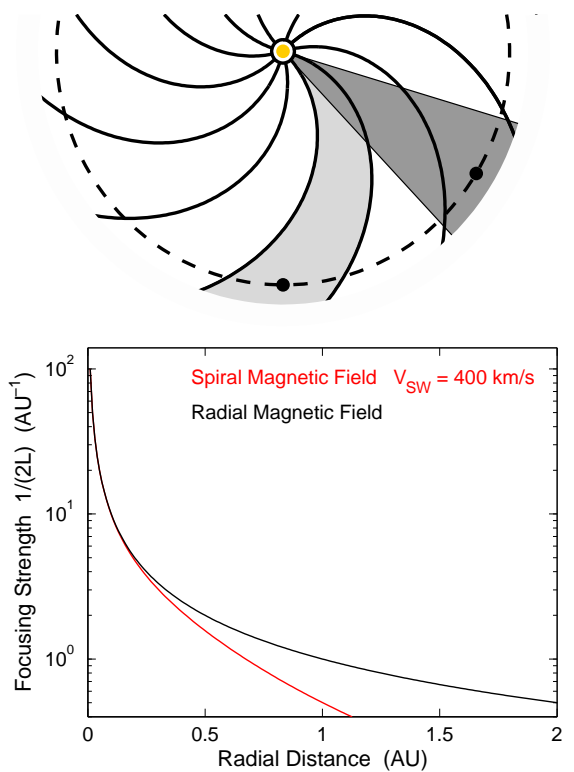

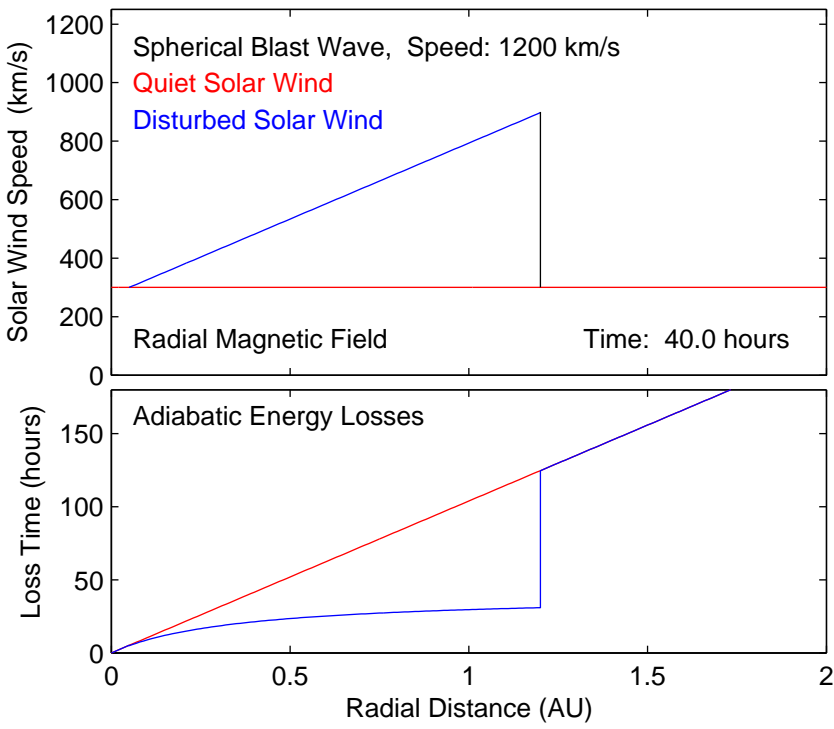

Figure 2: Upper left panel: sketch of the heliospheric magnetic field structure assumed in this work. Instead of propagating in spiral-shaped flux tubes in a Parker field (light grey) particles are considered to propagate in flux cones directed radially away from the Sun (dark grey). Lower left panel: focusing strength as a function radial distance for the above two geometries. Upper right panel: plasma speed profile at a parallel shock propagating in the solar wind with an assumed radial magnetic field. Lower right panel: adiabatic deceleration time scales in the quiet solar wind and in the expansion region behind the shock.

of equations 4.1 can be viewed as trajectories of quasi-particles, and the number of quasi-particles which are present in a volume element around a location $r$ during a time period from $t$ to $t+\Delta t$ is a measure for $I(r, \mu, E, t)$. The particles are traced in two reference frames: the co-moving solar wind frame, where the particles are scattered at magnetic fluctuations which are assumed to be static, and a stationary (with respect to the Sun) frame in which advection and focusing are considered. By obeying the proper transformations between the two above frames for the quasiparticle it is possible to take into account the effects of adiabatic losses in a diverging flow of scattering centers and of convection due to the isotropization of the particle distribution in the system moving with the solar wind. This method allows to include in a natural way the effect of first-order Fermi acceleration - the particles experience a strong adiabatic acceleration when they occasionally encounter the solar wind compression at the shock on their stochastic path, in contrast to their continuous small energy losses due to their coupling to the solar wind expansion.

In order to make full use of the information contained in angular dependence of observed particle fluxes, and for comparison with observations, we define an anisotropy parallel to the magnetic field

$$
A(r, t)=\frac{3 \int_{-1}^{+1} d \mu \mu f(r, \mu, t)}{\int_{-1}^{+1} d \mu f(r, \mu, t)}
$$

Figure 3 illustrates the results of a simulation for an impulsive injection at $t=0$ with $\sim E^{-4}$ and $V_{s h}=1200 \mathrm{Km} / \mathrm{s}, V_{0}=300 \mathrm{~km} / \mathrm{s}, V_{d}=900 \mathrm{~km} / \mathrm{s}$ and thus a compression ratio $R=3$, and spatially constant values of $\lambda_{r}=0.03 \mathrm{AU}$ (left side) and $\lambda_{r}=0.08 \mathrm{AU}$ (right side). The particle fluxes and anisotropies were determined in a frame which does not move with respect to the Sun, so 

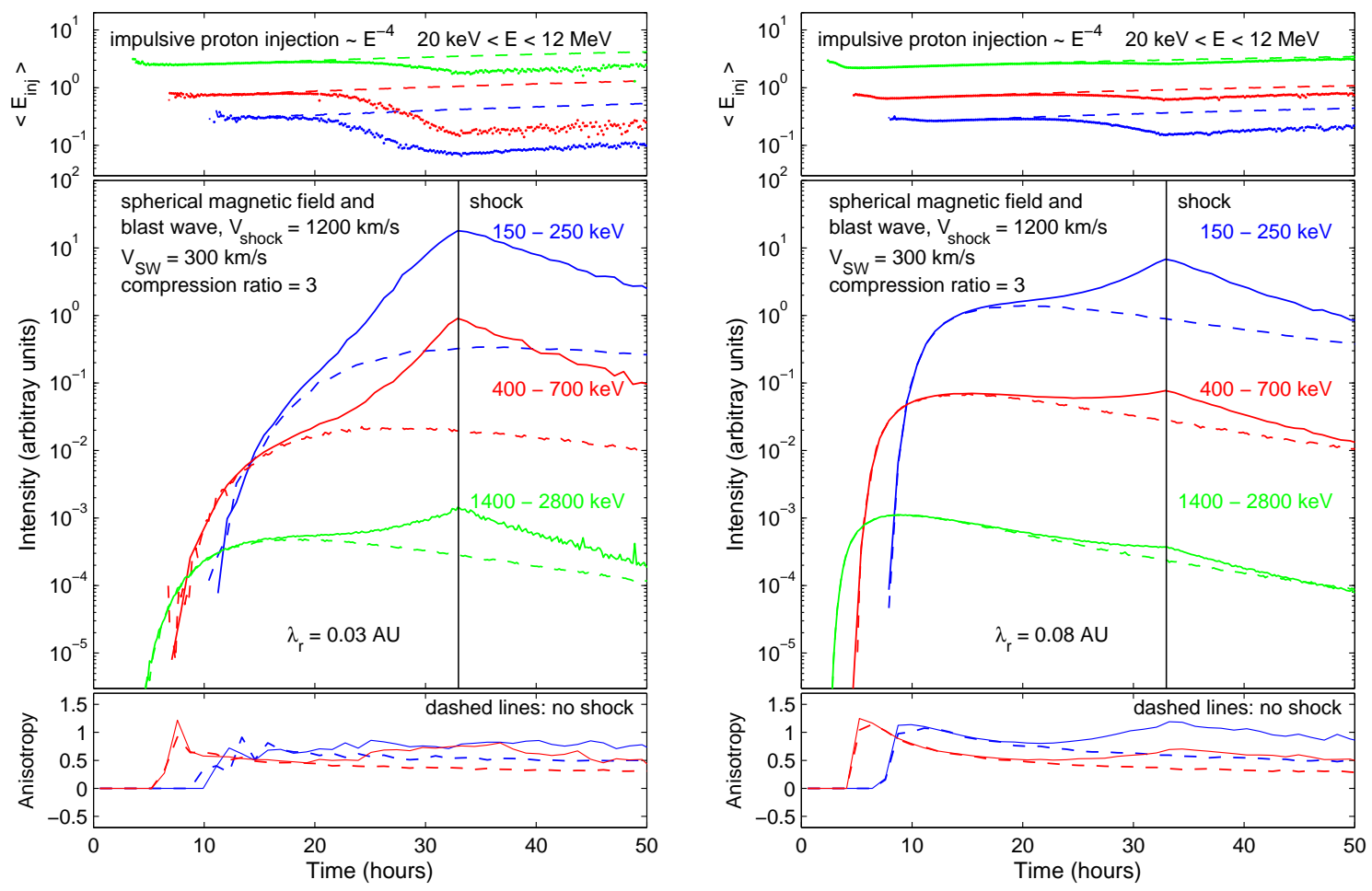

Figure 3: Time profiles of flare protons after interaction with with an interplanetary moving quasi parallel shock for $\lambda_{r}=0.03 \mathrm{AU}$ (left side) and $\lambda_{r}=0.08 \mathrm{AU}$ (right side). Particles are injected with an $\sim E^{-4}$ spectrum, $V_{s h}=1200 \mathrm{Km} / \mathrm{s}$, compression ratio $R=3, V_{0}=300 \mathrm{~km} / \mathrm{s}, V_{d}=900 \mathrm{~km} / \mathrm{s}$. The passage of the shock is marked by the vertical line in the middle panels. The upper panels show the average injection energy with which the protons in a given energy range are observed at a given time, the lower panels the anisotropy.

they would be therefore directly comparable with observations made on spacecraft moving with velocities which are small compared to that of the solar wind. The middle panel of the figure shows the time profiles of the proton fluxes in three energy ranges which were chosen to match those of the Wind 3DP/SST [15] particle detector. Solid lines indicate the results with the effects of the shock included, dashed lines the particle transport without a shock. The effect of the shock leads to an intensity maximum of the particle fluxes which is coincident with its passage, and a flux enhancement with respect to the solution without the shock by factors of 60, 40 and 5, respectively, for $\lambda_{r}=0.03 \mathrm{AU}$, and by factors of 6,3 and 1.6, respectively, for $\lambda_{r}=0.08 \mathrm{AU}$ in the three energy ranges in descending order. The lower panel of the figure shows that the anisotropies of the particles fluxes exhibit an increase at the passage of the shock, indicating that the shock acts as a moving source of particles. One of the advantages of the stochastic differential equation solver is that we can track the position and energy of the simulated particle as a function of space and time. In the upper panels of Figure 3 we have plotted the average energies with which the particles detected in each of the energy ranges had been injected close to the Sun. In the case of no shock we would expect the shown energy increase with time, which is explained by the fact that particles which were detected later had spent more time in the expanding solar wind and therefore lost more energy due to adiabatic deceleration. In the presence of a shock (solid lines) the injection energies decrease towards the passage of the shock which indicates that the particles observed around that time have been accelerated from lower energies by the shock. After the passage of the shock the injection 

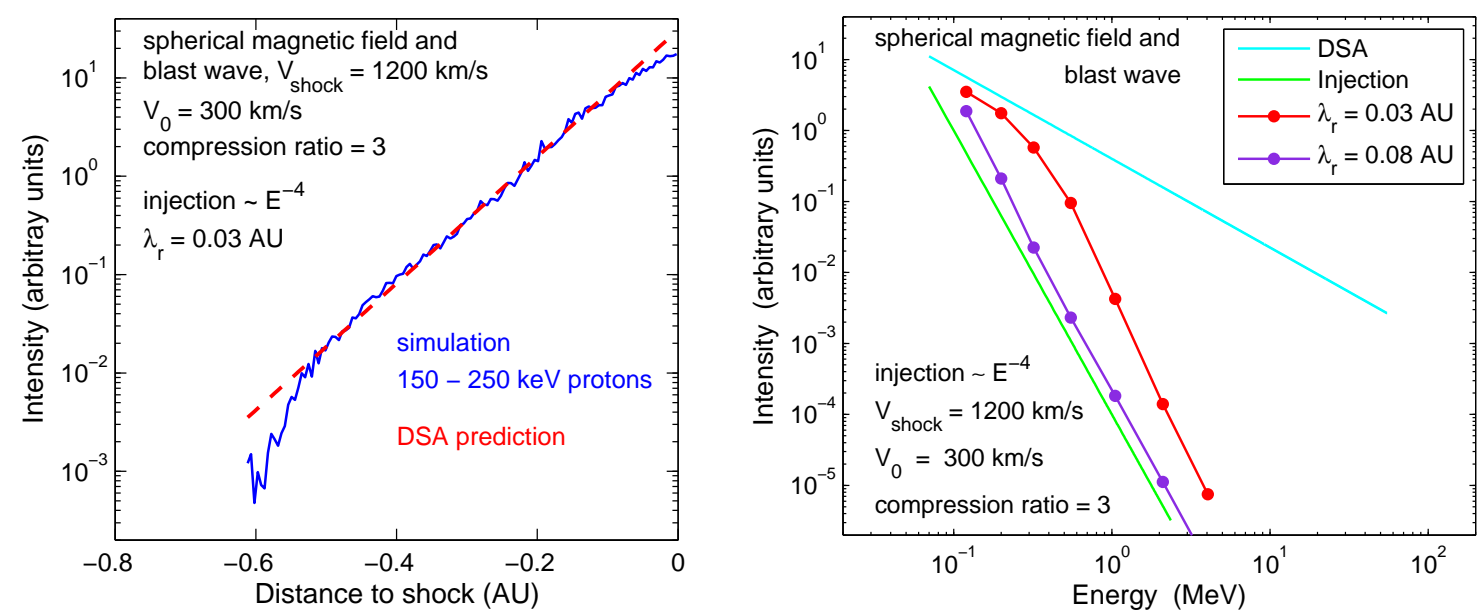

Figure 4: Left: Comparison of the intensity increase in front of the shock with the prediction from diffusive shock theory for $\lambda_{r}=0.03 \mathrm{AU}$. Right: Proton spectrum at the shock for $\lambda_{r}=0.03 \mathrm{AU}$ (red) and $\lambda_{r}=0.08$ $\mathrm{AU}$ (violet). Injection and solar wind parameters as in Fig. 3

energies increase faster as before which can be attributed to the fact that the over-expansion of the solar wind behind the shock leads to enhanced adiabatic deceleration.

Next we compare the results of our simulations with another prediction of DSA theory, e.g., the steady-state spatial dependence of the particle intensity in the upstream region as a function of the distance from the shock. We assume that at $1 \mathrm{AU}$ the curvature radius of the shock is large enough so that the geometry is approximately similar to that of the the one-dimensional case considered before and we can replace $r$ by $z$. In the shock's rest frame we have

$$
I(E, z) \propto \exp \left(\frac{U_{1} \cdot z}{K}\right) \propto \exp \left(\frac{3 U_{1} \cdot z}{\lambda v}\right)
$$

where $K=1 / 3 v \lambda$ is the spatial diffusion coefficient. Steady-state conditions are approximately reached after a characteristic time $t_{c} \sim\left(v \lambda_{u}\right) /\left(3 U^{2}\right)$ where $\lambda_{u}$ and $U$ are typical values of the mean free path and the flow speed in the upstream region. For the values considered here and $500 \mathrm{keV}$ protons we find $t_{c} \sim 7$ hours which is reasonably small enough compared to the shock travel time to $1 \mathrm{AU}$ of more than 30 hours, so that we can assume that a steady state in the particle transport is reached in the region upstream of the shock. With the kinematic parameters and radial distances involved we can convert the time axis in Figure 3 into a spatial axis (cf., [16]). The left side of Figure 4 shows a comparison of the proton intensity increases as a function of the distance to the shock obtained from the simulations with those predicted by DSA for $\lambda_{r}=0.03$ AU. We find an excellent agreement up to an upstream distance of $0.5 \mathrm{AU}$ from the shock. Deviations at larger distances are probably caused by the prompt component of the solar particles. At distances to the shock smaller than the scale of the assumed mean free paths we would not expect an exact match between solutions based on diffusion-convection equations (DSA) and those obtained based on kinetic equations as used in this work. In the right side of Figure 4 we compare the energy spectra at the time of the passage of the shock for $\lambda_{r}=0.03 \mathrm{AU}$ and $0.08 \mathrm{AU}$, respectively, with the injection spectrum and the predictions of DSA for the assumed shock parameters. As an important result we note that those spectra are steeper than the ones predicted by DSA for a quasi-parallel shock. Possible reasons for this finding will be discussed below. 


\section{Conclusions}

We have presented a model which simulates the transport and acceleration of solar particles injected at the Sun together with a traveling interplanetary shock for which we assume a simplified geometry in the form of a radial blast wave with a quasi-parallel magnetic structure. We find that for mean free paths of the order of $\lambda_{r}=0.05 \mathrm{AU}$ the results of our simulations can well reproduce observed intensity-time profiles of protons in the energy ranges of hundreds of $\mathrm{keV}$ to several $\mathrm{MeV}$ in interplanetary shock events, i.e., a rise to a first maximum after a few hours, followed by a plateau for a day or so, and then a second rise towards the shock. The spatial dependence of the intensity towards the shock in the upstream region is in good agreement with predictions from DSA, indicating that the spatial transport has reached an equilibrium. However, we find that the energy spectrum at the shock is considerably steeper as predicted by DSA for a given compression ratio. Although we cannot totally exclude the possibility that the comparatively low acceleration efficiency is caused partially by the simplistic shock model employed here our findings rather suggest that the effects of focusing, adiabatic deceleration in the expanding flow behind the shock and their variation in time and space prevent the acceleration process to reach a steady state, and an energy spectrum as predicted by DSA. More realistic assumptions about the solar wind structure, the angle between the shock normal and the magnetic field, and additional effects such as an enhanced particle scattering in the vicinity of the shock due to self-generated turbulence can be easily integrated into our simulation method and will be considered in future work.

This study was supported in part by GIF Grant no. 1145/2011. The authors benefited from discussions at a workshop on "Exploration of the inner Heliosphere", held at the International Space Science Institute (ISSI) in Bern, Switzerland.

\section{References}

[1] D. V. Reames, Space Sci. Rev 90 (1999) 413

[2] L. O. Drury, Rep. Prog. Phys. 46 (1983) 973

[3] J. A. le Roux, G. M. Webb, V. Florinski, G. P. Zank, ApJ 662 (2007) 350

[4] J. A. le Roux, G. M. Webb, ApJ 746 (2012) 104

[5] P. Zuo, M. Zhang, H. Rassoul, ApJ 767 (2013) 6

[6] C. K. Ng, L. J. Gleeson, Solar Phys. 20 (1971) 166

[7] P. A. Isenberg, JGRA 102 (1997) 4719

[8] C. W. Gardiner, Handbook of Stochastic Methods, Springer, Berlin 1983

[9] M. Zhang, G. Qin, H. Rassoul, ApJ 692 (2009) 109

[10] L. Kocharov, R. Vainio, G. A. Kovaltsov, J. Torsti, Solar Phys. 182 (1998) 195

[11] R. Vainio, L. Kocharov, T. Laitinen, ApJ 528 (2000) 1015

[12] W. Dröge, Y. Y. Kartavykh, B. Klecker, G. A. Kovaltsov, ApJ 709 (2010) 912

[13] J. R. Jokipii, ApJ 146 (1966) 480

[14] W. Dröge, Y. Y. Kartavykh, ApJ 693 (2009) 69

[15] R. P. Lin, et al., Space Sci. Rev 71 (1995) 125

[16] B. Klecker, M. Scholer, D. Hovestadt, G. Gloeckler, F. M. Ipavich, ApJ 215 (1981) 251 